\title{
Persistent currents in mesoscopic rings and boundary conformal field theory
}

\author{
S. Jaimungal, M.H.S. Amin and G. Rose \\ Department of Physics and Astronomy, University of British Columbia, Vancouver, BC V6T 1Z1, Canada
}

(June 11, 2018)

\begin{abstract}
A tight-binding model of electron dynamics in mesoscopic normal rings is studied using boundary conformal field theory. The partition function is calculated in the low energy limit and the persistent current generated as a function of an external magnetic flux threading the ring is found. We study the cases where there are defects and electron-electron interactions separately. The same temperature scaling for the persistent current is found in each case, and the functional form can be fitted, with a high degree of accuracy, to experimental data.

PACS numbers: 75.20.En, 05.30.Fk, 73.20.Dx
\end{abstract}

\section{INTRODUCTION}

It is well known that the topology of a condensed matter system can strongly influence its excitation spectrum. Consider for example the textbook case of a superconducting ring. If we write the order parameter in the form $\psi(x) \sim e^{i \Phi(x)}$ one can show that there will exist a set of topological excitations characterised by the winding number

$$
w=\frac{1}{2 \pi} \oint_{\Gamma} \vec{\nabla} \Phi \cdot d \vec{l}
$$

where $\Gamma$ represents a path encircling the hole in the ring. These excitations in general carry a current due to the non-zero gradient of the phase field in the ring. This effect is very well-studied [1], being fundamentally responsible for much of the physics of SQUIDs and related devices.

The prediction of the existence of this "persistent current" is, however, not contingent upon the substrate material being superconducting. Indeed it is apparent that the effect should be present in any system with the topology of a ring that permits excitations describable by field operators with the $U(1)$ symmetry alluded to above. Persistent currents in normal systems have in fact been observed-both in arrays of metal rings [2] and in individual metal [3] and semiconducting [4] rings. From a theoretical point of view this is not surprising because the persistent current is at heart an Aharonov-Bohm effect, independent of the actual nature of the excitations in the material.

This being said, it is quite obvious that the details of the supercurrent (such as its amplitude) will be strongly influenced by the details of the sample under investigation. For example, one expects that the lifetime of the excitations carrying the current will be of the order of the mean scattering time in the ring, which is heavily dependent upon the density of scatterers in the material and the details of the crystal field. If the associated mean free length is much less than the length of the ring $L$, then the effect will never be observed. Rigorous treatments of the effects of disorder on the persistent current have been performed both numerically [5] and analytically in restricted parameter regimes $[6$ 9].

In addition to the effects of impurities, it is suspected that the nature of the interaction between the supercurrent excitations can drastically affect the details of the resultant current 10 12]. For example, it has been shown that at zero temperature and in one dimension, the amplitude to backscatter from an impurity renormalizes to zero or to infinity for attractive or repulsive inter-quasiparticle interaction respectively [13].

In this paper we solve for the partition function of two different models; one with arbitrary short-range electronelectron interaction strength and no impurities, and one with an arbitrary number of impurity sites but no interaction between electrons. These calculations allow us to find the finite temperature persistent current developed in both cases, which can be directly compared to experiment [3]. We find the temperature dependence of both models, normalized to their zero temperature values for fixed external flux, follows a universal form which agrees quantitatively with experiment.

Our presentation is organised as follows. In section II we analyze the case of a defect-free ring threaded by a magnetic flux with short-range electron-electron Coulomb interactions. We demonstrate that this problem can be solved via the use of boundary conformal field theoretical techniques. In section III we consider a related model, where the Coulomb interactions have been removed and an arbitrary number of hopping defects added. It is shown that this new model can be mapped to one of quasiparticles on a defect-free ring, where the effective flux threading the ring is renormalized due to the presence of the defects in the bare model. In section IV we compare our findings to zero-temperature theoretical results and the finite-temperature data of Chandrasekhar et.al. [3] with which we find quantitative agreement. We conclude our presentation in section $\mathrm{V}$ with a summary of our results. 


\section{ANALYSIS OF DEFECT-FREE RING}

\section{A. Introduction of the Non-Interacting Model}

We begin our investigation by considering a tightbinding model in one dimension with constant hopping integrals $t_{0}$ and no electron-electron interaction. Throughout we shall choose a system of units such that $\hbar=1$ and $k_{B}=1$. We allow the ring to be threaded by a magnetic flux $\Phi=\int A_{\phi} d x=A_{\phi} L$, where $A_{\phi}$ is the component of the vector potential along the ring. The Hamiltonian for this model is

$$
H=t_{0} \sum_{j=1}^{N}\left\{e^{i \phi_{0} / N} \psi_{j+1}^{\dagger} \psi_{j}+\text { h.c. }\right\}
$$

with a periodic boundary condition $\psi_{N+1}=\psi_{1}$. Here we define $\phi_{0}=2 \pi \Phi / \Phi_{0}$ with $\Phi_{0}=2 \pi c / e$ the standard quantum of flux and $N$ the number of sites on the ring. We assume half-filling ( $N$ even); extension to the case of $N$ odd is straightforward.

\section{B. The Continuum Limit and Bosonization}

In this section we rewrite the model in a form that is accessible to analysis by conformal field theoretical techniques. Because conformal symmetry cannot exist in a lattice model, the first step is to rewrite (2) as a continuum model. We begin this procedure by first "opening" the ring and introducing an additional site at $j=N+1$. We then perform a gauge transformation

$$
\psi_{j} \rightarrow e^{i \phi_{0} j / N} \psi_{j}
$$

which allows (2) to be rewritten in the form

$$
H_{0}=t_{0} \sum_{j=1}^{N}\left\{\psi_{j+1}^{\dagger} \psi_{j}+\text { h.c. }\right\}
$$

In this case the periodic boundary conditions are changed to

$$
\psi_{N+1}=\kappa \psi_{1}
$$

where $\kappa=e^{-i \phi_{0}}$. We may now rewrite our model in terms of left and right moving fermions, namely $\psi(x)=$ $e^{i k_{F} x} \psi_{L}(x)+e^{-i k_{F} x} \psi_{R}(x)$, where $x=j a$ in the lattice model. Taking the continuum limit in the standard way gives

$$
H_{0}=\frac{v_{f}}{2 \pi} \int_{0}^{L} d x\left\{\psi_{L}^{\dagger}(x) i \frac{d}{d x} \psi_{L}(x)-\{L \leftrightarrow R\}\right\}
$$

which is exact up to terms of the order of the lattice spacing $a$. For ease of presentation we choose units such that the Fermi velocity $v_{f}=2 t_{0} a=1$. It is now possible to bosonize (6) by introducing a field $\Phi$ such that

$$
\psi_{L}(x) \sim e^{-i\left(\frac{\Phi}{2 r}+2 \pi r \widetilde{\Phi}\right)} \quad, \quad \psi_{R}(x) \sim e^{i\left(\frac{\Phi}{2 r}-2 \pi r \widetilde{\Phi}\right)}
$$

on the interval $0 \leq x \leq L$. Here $\widetilde{\Phi}=\Phi_{L}-\Phi_{R}$ is the field dual to $\Phi=\Phi_{L}+\Phi_{R}$, and $r$ denotes the compactification radius of the bosonic field $\Phi \sim \Phi+2 \pi r$. In the noninteracting case under consideration $r$ is taken to be at its self-dual point $r=\frac{1}{\sqrt{4 \pi}}$. Note that it is not necessary to include Klein factors [14] here as the number of left and right moving fermions is conserved in this system. Substitution of (7) into the boundary condition (5) gives

$$
\widetilde{\Phi}(L)-\widetilde{\Phi}(0)=\frac{\phi_{0}}{2 \pi r} \quad, \quad \Phi(L)+\Phi(0)=0
$$

We introduce boundaries into the model by folding the ring in half. Mathematically this is achieved by introducing two bosonic field degrees of freedom defined on the half interval

$$
\Phi_{e, o}(x)=\Phi(L-x) \pm \Phi(x) \quad, \quad 0 \leq x \leq L / 2
$$

In terms of the fields $\Phi_{e, o}(x)$ the boundary conditions (8) become

$$
\widetilde{\Phi}_{o}(0)=\frac{\phi_{0}}{2 \pi r} \quad, \quad \Phi_{e}(0)=0
$$

and the Hamiltonian becomes

$$
H_{0}=\frac{1}{2 \pi} \int_{0}^{L / 2} d x\left\{\left(\frac{d \Phi_{e}}{d x}\right)^{2}+\left(\frac{d \Phi_{o}}{d x}\right)^{2}\right\}
$$

We have reduced the boundary conditions on the fermions (5) to simple Dirichlet and Neumann conditions on the even and odd bosons while maintaining the free nature of the Hamiltonian.

\section{The Role of Conformal Invariance}

Thus far we have translated our original problem of a perfect ring threaded by a flux to one of two free bosons on the half-interval $[0, L / 2]$ with boundary conditions (10). The partition function for such a system is immediately available via the use of boundary conformal field theory techniques [15]. In this section we shall present an overview of the relevant conformal machinery and compute the partition function for our model (2).

In a conformally invariant theory, a conformal boundary condition is given by those states which satisfy the condition

$$
\left.(T-\bar{T})\right|_{b d .}|B\rangle=0
$$

where $T$ and $\bar{T}$ denote the holomorphic and antiholomorphic components of the energy momentum tensor respectively. This condition corresponds to the physical statement that no energy is allowed to flow off of the 
edges of the system. The general state satisfying the above condition is an Ishibashi state [16],

$$
|\mu\rangle\rangle \equiv \sum_{N}|\mu, N\rangle \otimes \overline{|\mu, N\rangle}
$$

where the sum is over all the descendents of the heighest weight state defined by $|\mu\rangle$. In the case of a single free boson, with compactification radius $r$, one can construct states which not only satisfy the reflection condition but are also eigenvectors of the field or its dual (with eigenvalue $\left.\varphi_{0}\right)$ at the boundary 115,

$$
\begin{aligned}
& \left|D\left(\varphi_{0}\right)\right\rangle=\frac{1}{\sqrt{2 r}} \sum_{k=-\infty}^{\infty} e^{-i k \varphi_{0} / r} \exp \left\{-\sum_{n=1}^{\infty} a_{n}^{\dagger} \tilde{a}_{n}^{\dagger}\right\}|(0, k)\rangle \\
& \left|N\left(\varphi_{0}\right)\right\rangle=\sqrt{r} \sum_{w=-\infty}^{\infty} e^{-2 i r w \varphi_{0}} \exp \left\{+\sum_{n=1}^{\infty} a_{n}^{\dagger} \tilde{a}_{n}^{\dagger}\right\}|(w, 0)\rangle
\end{aligned}
$$

These states correspond to Dirichlet and Neumann states respectively. The details of the various operators appearing here can be found in 115 . It is sufficient to note that $|(w, k)\rangle$ denotes a Fock vacuum with $a_{0}$ eigenvalue $k$ and $\tilde{a}_{0}$ eigenvalue $w$ and $a_{n}, \tilde{a}_{n}$ are the usual annihilation operators which make up the bosonic field in canonical quantization.

It is well known that one can compute the partition function of a conformal field theory with boundary in two equivalent ways 17, 18]: $Z=\operatorname{Tre}^{-\beta H_{a b}}$ where $H_{a b}$ denotes the Hamiltonian which respects the boundary conditions $a$ and $b$ on either end of the system, or through a modular transformation, which interchanges space and time directions, as $Z=\left\langle a\left|e^{-L H_{\beta}}\right| b\right\rangle$ where $H_{\beta}$ denotes the Hamiltonian on a circle of radius $\beta$. With this in mind, the single free boson has four possible partition functions corresponding to the four possible boundary conditions $D D, N N, D N$ or $N D$. These have all been computed in [15] and are given by

$$
\begin{aligned}
Z_{D D} & =<D\left(\varphi_{0}\right)\left|e^{-\frac{1}{2} H_{\beta}}\right| D\left(\varphi_{0}^{\prime}\right)> \\
& =\frac{1}{\eta(q)} q^{-\left(\Delta \varphi_{0} / \pi\right)^{2}} \vartheta_{3}\left(-2 i r \Delta \varphi_{0} \beta ; q^{2 r^{2}}\right) \\
Z_{N N} & =<N\left(\varphi_{0}\right)\left|e^{-\frac{1}{2} H_{\beta}}\right| N\left(\varphi_{0}^{\prime}\right)> \\
& =\frac{1}{\eta(q)} q^{-\left(\Delta \varphi_{0} / \pi\right)^{2}} \vartheta_{3}\left(-i \Delta \varphi_{0} \beta / r ; q^{1 / 2 r^{2}}\right) \\
Z_{D N} & =<D\left(\varphi_{0}\right)\left|e^{-\frac{1}{2} H_{\beta}}\right| N\left(\varphi_{0}^{\prime}\right)>=\frac{1}{2 \eta(q)} \vartheta_{2}(0 ; q)
\end{aligned}
$$

where $\Delta \varphi_{0}=\varphi_{0}-\varphi_{0}^{\prime}$ and $q=e^{-2 \pi \beta}$ (The length scale $L$ has been absorbed in the definition of $\beta$, i.e. to restore the length scales $\beta \rightarrow \beta / L) . \eta(q)$ and $\vartheta_{2,3}(w, q)$ denote the Dedekind eta function and Jacobi theta functions respectively. Explicitly these are

$$
\eta(q)=q^{1 / 24} \prod_{n=1}^{\infty}\left(1-q^{n}\right)
$$

$$
\begin{aligned}
& \vartheta_{2}(w, q)=2 q^{1 / 4} \sum_{n=0}^{\infty} q^{n(n+1)} \cos (2 n+1) \omega \\
& \vartheta_{3}(w, q)=1+2 \sum_{n=1}^{\infty} q^{n^{2}} \cos 2 n \omega
\end{aligned}
$$

\section{Calculation of the Partition Function}

It is now a straightforward matter to apply these results to the free Hamiltonian for even and odd bosons with boundary conditions (10). Since the two bosons are non-interacting the boundary states associated with them are tensor products of the single boson boundary state. Therefore we can write the relevant partition function as

$$
\begin{aligned}
Z & =\left\langle D(0)\left|\otimes\left\langle N\left(\phi_{0} / 2 \pi r\right)\left|e^{-\frac{1}{2}\left(H_{\beta}^{e}+H_{\beta}^{o}\right)}\right| N(0)\right\rangle \otimes\right| D(0)\right\rangle \\
& =\left\langle D(0)\left|e^{-\frac{1}{2} H_{\beta}^{e}}\right| D(0)\right\rangle\left\langle N\left(\phi_{0} / 2 \pi r\right)\left|e^{-\frac{1}{2} H_{\beta}^{o}}\right| N(0)\right\rangle \\
& =\frac{q^{-\left(\phi_{0} / 2 \pi^{2} r\right)^{2}}}{\eta^{2}(q)} \vartheta_{3}\left(0 ; q^{r^{2} / 2}\right) \vartheta_{3}\left(-\frac{i \phi_{0} \beta}{\pi r^{2}} ; q^{1 / 8 r^{2}}\right)
\end{aligned}
$$

Notice that we have chosen the boundary state at $x=$ $L / 2$ to be $\widetilde{\Phi}_{o}=0$ and $\Phi_{e}=0$. One can be convinced that this is correct by starting with the condition (5) where the sites $N+1$ and 1 are replaced by $N / 2+1$ and $N / 2$ respectively and taking $\kappa=1$. This effectively sets the flux in (10) to zero.

\section{E. Inclusion of Coulomb Interactions}

This completes the analysis of our original toy model (2). Let us now consider what happens when we include short-range Coulomb interactions. We repeat the procedure followed in the previous sections with the Hamiltonian

$$
\begin{aligned}
H= & -t_{0} \sum_{j=1}^{N}\left\{e^{i \phi_{0} / N} \psi_{j+1}^{\dagger} \psi_{j}+h . c .\right\} \\
& +U_{0} \sum_{j} \psi^{\dagger}(j) \psi(j) \psi^{\dagger}(j+1) \psi(j+1)
\end{aligned}
$$

It is well known 19 that the only effect of the Coulomb term is to renormalize both the radius of compactification

$$
r=\frac{1}{\sqrt{4 \pi}} \sqrt{1+\frac{U_{0}}{t_{0} \pi}}
$$

and the Fermi velocity

$$
v_{f}=2 t_{0} a\left(1+\frac{U_{0}}{2 \pi t_{0}}\right)
$$


In order to accomodate this we choose new units such that $v_{f}=1$. It is then straightforward to use (12) to calculate the free energy of the system and then the persistent current as a function of the compactification radius $r\left(U_{0}\right)$. Explicitly this is

$$
J=-\frac{e}{\beta} \frac{\partial \ln Z}{\partial \phi_{0}}
$$

We show in fig.1 the results of this calculation. Varying the parameter $r$ does not qualitatively affect the shape of $J\left(\phi_{0}, \beta\right)$.

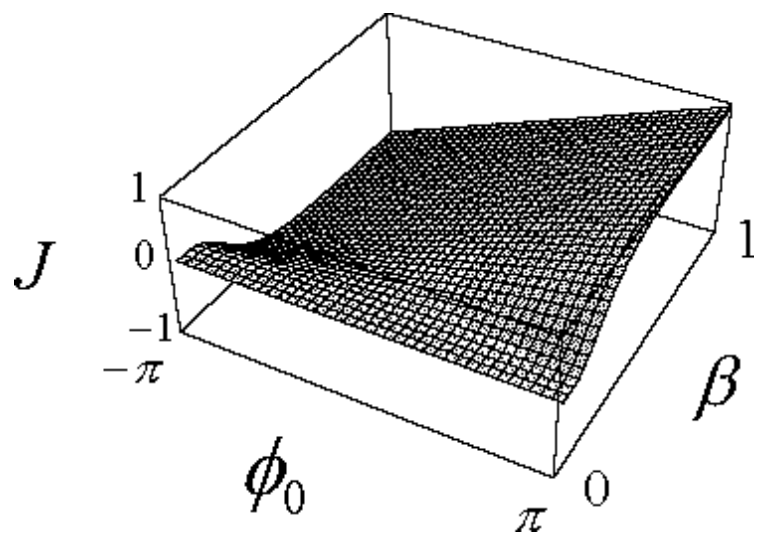

FIG. 1. Persistent current in a defect-free ring for arbitrary Coulomb interaction strength (units for $\beta$ are chosen to highlight the transition from the high temperature regime to the low temperature limit (corresponding here to $\beta \sim 1$ ) and units for $J$ are arbitrary). Note that $J$ is periodic in $\phi_{0}$ with period $2 \pi$.

\section{INCLUSION OF HOPPING DEFECTS}

We now demonstrate how this analysis may be extended to deal with the presence of hopping defects. The Hamiltonian for a ring with $m$ defects reads, after an appropriate gauge transformation,

$$
\begin{aligned}
H= & -t_{0} \sum_{j \notin A}\left\{\psi^{\dagger}(j+1) \psi(j)+\text { h.c. }\right\} \\
& -t_{0} \sum_{j \in A}\left\{\kappa_{j} \psi^{\dagger}(j) \psi(j+1)+\text { h.c. }\right\}
\end{aligned}
$$

Here the set of sites $\{A\}$ labels the various defect links and $\kappa_{i}=\rho_{i} e^{-i \phi_{0} / m}$ denotes the strengths of the defects. It is a straightforward matter to diagonalize (17);

$$
H_{0}=-\sum_{k: f(k)=0} \epsilon_{k} \eta_{k}^{\dagger} \eta_{k}
$$

The dispersion relation here is $\epsilon_{k} \equiv 2 t_{0} \cos (k)$, and the restriction to $f(k)=0$ implements the quantization condition. Note that in all that follows we will only be interested in energies very close to the Fermi surface. We shall assume that if there are any localized states, their energies are far from $E_{f}$ and their contribution to the low-energy physics can be neglected.

As an explicit example of the quantization condition, one finds for the case of a single defect $f(k)=0$ reduces to 20]:

$$
\begin{aligned}
f_{1}(k) & =\rho^{2} \sin (k(N-1)) \\
& +2 \rho \cos \left(\phi_{0}\right) \sin (k)-\sin (k(N+1))=0
\end{aligned}
$$

In the low-energy limit under investigation, for an arbitrary number of defects, the quantization condition may be linearized near the Fermi points $k= \pm \frac{\pi}{2}$. Performing this procedure, one finds that the effects of the defects and flux may be subsumed into a shift in the fermionic spectrum given by

$$
k= \pm \frac{\pi}{2}+\frac{2 \pi}{N} n+\frac{\alpha\left(\{\kappa\}, \phi_{0}\right)}{N} \quad, \quad n \in \mathbb{Z} .
$$

For the single defect case (19) one can show 20 that $\alpha$ is given explicitly by

$$
\alpha=\cos ^{-1}\left(\frac{2 \rho}{1+\rho^{2}} \cos \phi_{0}\right)
$$

In general for $m$ defects $\alpha$ is found by solving a secular determinant of order $2^{m}$.

Introducing new quasi-particle fermionic operators

$$
\widetilde{\psi}(j)=\sum_{n} e^{i \frac{2 \pi}{N} n j} \eta_{n}
$$

into our Hamiltonian (18) we find that

$$
H=-t_{0} \sum_{j=1}^{N}\left\{e^{i \alpha / N} \widetilde{\psi}^{\dagger}(j+1) \widetilde{\psi}(j)+\text { h.c. }\right\}
$$

which is the original toy model (2) with an effective flux given by $\alpha$ instead of $\phi_{0}$. This demonstrates that the low energy limit of the multi-defect case can be reduced to one of free quasi-particles on a ring threaded by an effective flux $\alpha\left(\{\kappa\}, \phi_{0}\right)$. At this point one can apply the analysis of the previous section to obtain the partition function and therefore the persistent current. Shown in fig. 2 is the persistent current in the simplest case where we have only one defect. We find that the flux dependence of $J$ changes dramatically as $\rho$ departs from 1, evolving from the sawtooth shape seen in the no-defect case to a sinusoidal variation.

\section{COMPARISON OF TEMPERATURE DEPENDENCE OF PERSISTENT CURRENT TO EXPERIMENT}

We have calculated the partition functions for both models under consideration. This allows the temperature dependence of the persistent current to be extracted for 


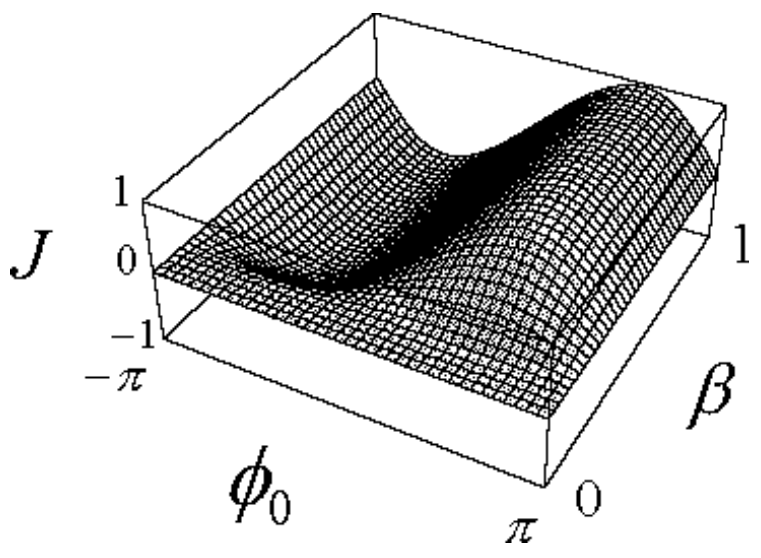

FIG. 2. Persistent current developed in a ring with no electron-electron interaction and one hopping impurity with strength $\rho=6$. Units for $J$ and $\beta$ are the same as those defined in figure 1.

each. We may perform both calculations in parallel by retaining all factors of $r$ and $\alpha$ in our expressions, keeping in mind that these cannot be varied independently. For the defect-free model, $\alpha=\phi_{0}$ and $r$ is arbitrary. For the non-interacting model, $\alpha$ is given by solving for the roots of the quantization condition $f(k)=0$ (18) linearized near the Fermi points and $r=1 / \sqrt{4 \pi}$.

With this caveat, the free energy can be written in general as

$$
\begin{aligned}
F & =-\frac{1}{\beta} \ln Z=\frac{\alpha^{2}}{2 \pi^{3} r^{2}}-\frac{1}{\beta}\left(\ln \vartheta_{3}\left(0 ; e^{-\pi \beta r^{2}}\right)\right. \\
& \left.+\ln \vartheta_{3}\left(-\frac{i \alpha \beta}{\pi r^{2}} ; e^{-4 \pi \beta / r^{2}}\right)-2 \ln \eta(q)\right)
\end{aligned}
$$

with subsequent persistent current

$$
J=-c \frac{\partial F}{\partial \vec{A}}=-e \frac{\partial \alpha}{\partial \phi_{0}} \frac{\partial F}{\partial \alpha}
$$

whose zero temperature limit is simply

$$
J_{0}=-\frac{e \alpha}{\pi^{3} r^{2}} \frac{\partial \alpha}{\partial \phi_{0}}
$$

If we normalize the persistent current to this value we find that

$$
\frac{J}{J_{0}}=1-\frac{i \pi^{2}}{\alpha} \frac{\vartheta_{3}^{\prime}}{\vartheta_{3}}\left(\frac{i \alpha T_{1}}{2 \pi^{2} T}, e^{-T_{1} / T}\right)
$$

where we have introduced the energy scale

$$
T_{1}=\frac{4 v_{f}}{L r^{2}}
$$

Note that though $\alpha$ can be a complicated function of $\phi_{0}$ and the defects, it is bounded, being defined in our model on the region $[-\pi . . \pi]$. It is interesting to note that the form of (26) is not a strong function of $\alpha$, its shape nearly entirely dependent on the value of $T_{1}$ (see fig.3). This means that regardless of the model chosen, this one parameter (which can be varied arbitrarily in both cases) determines the temperature dependence of the normalized persistent current for fixed $\phi_{0}$, regardless of the resultant value of $\alpha$.

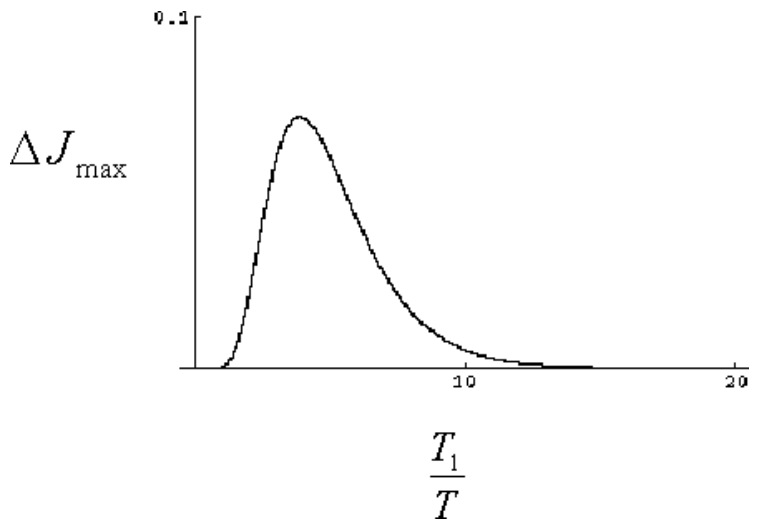

FIG. 3. $\Delta J_{\max }$ is the maximum variation in $J / J_{0}$ for $\alpha \in[-\pi . . \pi]$, here given as a function of $T_{1} / T$. We see that $\Delta J_{\max }<<1$ for all $T_{1} / T$, demonstrating the weak dependence of $J / J_{0}$ on $\alpha$.

We now compare the form derived for $J / J_{0}$ to the experimental results of Chandrasekhar et.al. [3] obtained from mesoscopic gold rings. In these experiments the authors present data for the maximal normalized persistent current, defined to be the solution of (26) with $\phi_{0}$ chosen so as to maximize the zero to peak amplitude of the signal. Shown in fig.(14) are the results of a fit to the data. The circles represent values gathered from a $1.4 \mu m \times 2.6 \mu m$ loop and the squares the response from a $2.4 \mu \mathrm{m}$ ring. We find that the value $T_{1}=0.09 \mathrm{~K}$ fits both cases extremely well. This finding lends credence to our claim that the details of the defects do not affect the temperature dependence of $J / J_{0}$. This is simply because the two samples considered in the experiments must have had differing defect structures (corresponding in our language to different shifts $\alpha$ ) yet the experiments show the same temperature dependence.

\section{CONCLUSIONS}

Using boundary conformal field theoretical techniques we have computed the partition function of a tightbinding model of electron dynamics in a mesoscopic normal ring. Defects and electron-electron interactions were introduced separately. In the case where defects are introduced, we mapped the problem onto a defect-free 


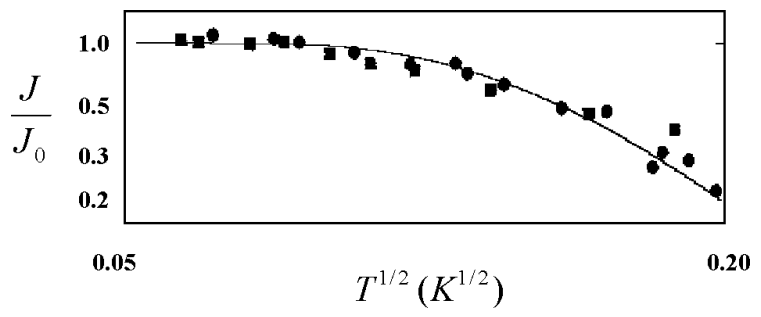

FIG. 4. Fit to the data of Chandrasekhar et.al. [3] with $T_{1}=0.09 \mathrm{~K}$.

model with an effective flux which depends on the details of the original defects. The second case, where electronelectron interactions are included within the defect-free model, was solved by noting that such interactions serve only to alter the compactification radius of the bosons and the effective Fermi velocity.

Using the partition functions we computed the persistent current for both cases as a function of flux and temperature. The model with defects generated a persistent current that was found to change its flux dependence from a sawtooth to a sinusoidal shape even for one weakly scattering impurity. Futhermore, the amplitude of the oscillations decreased as the strength of the defect increased. However, the inclusion of electronelectron interactions in the defect-free case produces a different behaviour: the sawtooth nature is not altered and the amplitude of the current decreases only very weakly as the interaction strength is increased. Although the functional dependence on flux differs in the two cases described here, their normalized currents have identical temperature dependence. There is one single free parameter in the expression for the current which sets the scale in which temperature is measured. This suggests that such a functional dependence on temperature is generic in the sense that the microscopic details of the interactions and impurities do not affect it when taken separately.

We compared the temperature dependence with experiment [3] and with only one fitting parameter (the energy scale) found excellent agreement. In such an experimental setup one expects that both electron-electron interactions and defects play a significant role, yet the predictions of our simple model were able to capture, quantitatively, those results. This seems to indicate that the results obtained for the normalized persistent current are not sensitive to any interplay between defects and electron-electron interactions in the bare model-that is, including defects and electron-electron interactions simultaneously should lead to analogous predictions.

\section{ACKNOWLEDGMENTS}

We would like to thank I. Affleck, P. Stamp, A. Zagoskin, J.S. Caux, C.L. Kane and Kurgan for helpful discussions and N. Prokofi'ev for correspondence. This work was supported, in part, by the University of British Columbia Graduate Fellowship program and by NSERC.

[1] V. Kashurnikov, A. Podlivaev, N.V. Prokof'ev and B. Svistunov, Phys. Rev. B53, 13091 (1996).

[2] L.P. Levy, G. Dolan, J. Dunsmuir, and H. Bouchiat, Phys. Rev. Lett. 64, 2074 (1990).

[3] V. Chandrasekhar, R.A. Webb, M.J. Brady, M.B. Ketchen, W.J. Gallagher, and A. Kleinsasser, Phys. Rev. Lett. 67, 3578 (1991).

[4] D. Mailly, C. Chapelier, and A. Benoit, Phys. Rev. Lett. 70, 2020 (1993).

[5] G. Bouzerar, D. Poilblanc, and G. Montambaux, Phys. Rev. B49, 8258 (1994).

[6] A.O. Gogolin and N.V. Prokof'ev, Phys. Rev. B50, 4921 (1994).

[7] H.F. Cheung, E.K. Riedel, and Y. Gefen, Phys. Rev. Lett. 62, 587 (1989).

[8] R. Landauer and M. Buttiker, Phys. Rev. Lett. 54, 2049 (1985).

[9] Jia-Lin Zhu, Xi Chen and Yoshiyuki Kawazoe, Phys. Rev. B 55, 16300 (1997).

[10] V. Ambegaokar and U. Eckern, Phys. Rev. Lett. 65, 381 (1990).

[11] B. Altshuler, Y. Gefen, and Y. Imry, Phys. Rev. Lett. 66, 88 (1991).

[12] H. Bouchiat and G. Montambaux, J. Phys. (Paris) 50, 2695 (1989).

[13] C. L. Kane and M. P. A. Fisher, Phys. Rev. B 46, 15233 (1992).

[14] Jan von Delft and H. Schoeller, Annalen der Physik 4, 225 (1998).

[15] M. Oshikawa and I. Affleck, Nucl. Phys. B495, 533 (1997).

[16] N. Ishibashi, Mod. Phys. Lett. A4, 251 (1989).

[17] J. L. Cardy, Nucl. Phys. B275, 200 (1986).

[18] J. L. Cardy, Nucl. Phys. B324, 584 (1989).

[19] I. Affleck, Lecture notes, Les Houches summer school, in Fields, Strings and Critical Phenomena, (1998).

[20] M. Henkel and D. Karevski, Eur. Phys. J B5, 787 (1998). 